\title{
Consolidated bioprocessing of lignocellulose for production of glucaric acid by an artificial microbial consortium
}

\section{Chaofeng Li}

Northwest A\&F University

Xiaofeng Lin

Northwest A\&F University

Xing Ling

Northwest A\&F University

Shuo Li

Northwest A\&F University

Hao Fang ( $\square$ fanghao@nwsuaf.edu.cn )

Northwest A\&F University https://orcid.org/0000-0002-2445-4993

\section{Research}

Keywords: Consolidated bioprocessing (CBP); D-glucaric acid; Lignocellulose; Microbial consortium; Trichoderma reesei; Saccharomyces cerevisiae

Posted Date: January 28th, 2021

DOl: https://doi.org/10.21203/rs.3.rs-29885/v2

License: (c) (1) This work is licensed under a Creative Commons Attribution 4.0 International License. Read Full License

Version of Record: A version of this preprint was published at Biotechnology for Biofuels on April 30th, 2021. See the published version at https://doi.org/10.1186/s13068-021-01961-7. 


\section{Abstract}

Background: The biomanufacturing of D-glucaric acid has been attracted increasing interest because it is one of the top value-added chemicals produced from biomass. Saccharomyces cerevisiae is regarded as an excellent host for D-glucaric acid production.

Results: The opi1 gene was knocked out because of its negative regulation on myo-inositol synthesis, which is the limiting step of D-glucaric acid production by S. cerevisiae. Then we constructed the biosynthetic pathway of D-glucaric acid in S. cerevisiae INVSc1 opi1 $\Delta$ and obtained two engineered strains, LGA-1 and LGA-C, producing record breaking titers of D-glucaric acid, $9.53 \pm 0.46 \mathrm{~g} / \mathrm{L}$ and $11.21 \pm$ $0.63 \mathrm{~g} / \mathrm{L}$ D-glucaric acid from $30 \mathrm{~g} / \mathrm{L}$ glucose and $10.8 \mathrm{~g} / \mathrm{L}$ myo-inositol in the mode of fed-batch fermentation, respectively. However, LGA-1 was more preferable because of the genetic stability and the outperformance in applications. So far, there have been no reports on D-glucaric acid production from lignocellulose. Therefore, the biorefinery processes including separated hydrolysis and fermentation (SHF), simultaneous saccharification and fermentation (SSF) and consolidated bioprocessing (CBP) were investigated and compared. CBP by an artificial microbial consortium composed of Trichoderma reesei Rut-C30 and S. cerevisiae LGA-1 was found to have relatively high D-glucaric acid titers and yields after 7 $\mathrm{d}$ fermentation, $0.54 \pm 0.12 \mathrm{~g} / \mathrm{L}$ D-glucaric acid from $15 \mathrm{~g} / \mathrm{L}$ Avicel, and $0.45 \pm 0.06 \mathrm{~g} / \mathrm{L}$ D-glucaric acid from $15 \mathrm{~g} / \mathrm{L}$ steam exploded corn stover (SECS), respectively. In attempts to design the microbial consortium for more efficient CBP the team consisted of the two members, T. reesei Rut-C30 and $S$. cerevisiae LGA-1, was found to be the best with excellent work distribution and collaboration.

Conclusions: Two engineered S. cerevisiae strains, LGA-1 and LGA-C, with high titers of D-glucaric acid were obtained. This indicates that $S$. cerevisiae INVSc1 was an excellent host. Lignocellulose is a more preferable substrate than myo-inositol. SHF, SSF and CBP were studied and CBP by an artificial microbial consortium of $T$. reesei Rut-C30 and S. cerevisiae LGA-1 was found to be promising because of the relatively high titer and yield. T. reesei Rut-C30 and S. cerevisiae LGA-1 were proved to be the best teammates for CBP. Further work should be done to improve the efficiency of this microbial consortium for D-glucaric acid production from lignocellulose.

\section{Background}

D-Glucaric acid, identified as "top value-added chemical from biomass" by US Department of Energy in 2004 [1], is an important platform chemical with a wide variety of applications such as therapeutic uses and biopolymer production [2-4]. Conventionally, D-glucaric acid was produced via nitric acid oxidation of D-glucose. It is a nonselective and expensive process associated with a large exotherm, low yields and toxic byproducts [4-6]. Biological production of D-glucaric acid has been attracted increasing interest due to the potential for a cheaper and more environmentally friendly process by avoiding costly catalysts and harsh reaction conditions $[3,4]$. 
A biosynthetic route from D-glucose to D-glucaric acid consisting of three heterologous genes was constructed in recombinant Escherichia coli by Moon et al.[2]. They were myo-inositol-1-phosphate synthase (Ino1) from Saccharomyces cerevisiae, myo-inositol oxygenase (MIOX) from Mus musculus and urinate dehydrogenase (UDH) from Pseudomonas syringae. But the titer of D-glucaric acid was low, only $0.72 \mathrm{~g} / \mathrm{L}$ [2]. After optimizing the induction and culture conditions, a slight increase in D-glucaric acid titer to $1.13 \mathrm{~g} / \mathrm{L}$ was achieved $[2,4]$. MIOX was found to be the rate-limiting step of the biosynthetic route for such low titers [2]. A synthetic scaffold was used to increase MIOX stability and the efficiency of the biosynthetic route, leading to $2.5 \mathrm{~g} / \mathrm{L}$ D-glucaric acid from $10 \mathrm{~g} / \mathrm{L}$ D-glucose [7]. Moreover, an N-terminal SUMO fusion to MIOX gave rise to a $75 \%$ increase in D-glucaric acid production from myo-inositol. Up to $4.85 \mathrm{~g} / \mathrm{L}$ of D-glucaric acid was produced from $10.8 \mathrm{~g} / \mathrm{L}$ myo-inositol in recombinant $E$. coli [4]. However, $E$. coli is thought to be not suitable for D-glucaric acid production at high titer because D-glucaric acid concentrations above $5 \mathrm{~g} / \mathrm{L}$ appears to inhibit its further production by $E$. colithrough a $\mathrm{pH}$-mediated effect $[2,3,5]$.

Gupta et al.[5] ported the synthetic D-glucaric acid pathway from E. coli to S. cerevisiae, another model strain widely used industry that has better acid tolerance [3]. They found that MIOX4 from Arabidopsis thaliana outperformed MIOX from M. musculus [5]. The maximal titer of the S. cerevisiae strain with MIOX4 was $1.6 \mathrm{~g} / \mathrm{L} \mathrm{D-glucaric} \mathrm{acid} \mathrm{from} \mathrm{glucose} \mathrm{supplemented} \mathrm{with} \mathrm{myo-inositol} \mathrm{[5].} \mathrm{Chen} \mathrm{et} \mathrm{al.}$ [3] adopted delta-sequence-based integrative expression to increase MIOX4 activity and stability, successfully increasing glucaric acid titer about eight times over that of episomal expression. Combining this strategy with fed-batch fermentation supplemented with $60 \mathrm{mM}(10.8 \mathrm{~g} / \mathrm{L})$ myo-inositol, a titer of 6 $\mathrm{g} / \mathrm{L}(28.6 \mathrm{mM}) \mathrm{D}$-glucaric acid was achieved. This was the highest had been ever reported in $S$. cerevisiae [3].

Here we used the same genes and strategy as Chen et al. reported [3] to construct the biosynthetic route for D-glucaric acid production in a different baker's yeast strain, S. cerevisiae INVSc1. In light of myoinositol availability was rate-limiting in the $S$. cerevisiae strain with the miox4 gene from $A$. thaliana [3, 5], the opi1 gene in $S$. cerevisiae INVSc1 was also knocked out accordingly to remove its negative regulation on myo-inositol synthesis [3, 8]. As a result, a more robust engineered strain of $S$. cerevisiae INVSc1 was obtained, producing the record-breaking titer of D-glucaric acid in S. cerevisiae. As the top value-added chemical from biomass, however, there have been no reports on D-glucaric acid production from lignocellulose in the scenario of biorefinery.

We applied the engineered $S$. cerevisiae strain to bioprocesses for D-glucaric acid production from model cellulose and natural lignocelluloses, the same as biofuel production such as bioethanol. Fig. 1 illustrates the biorefinery processes including separated hydrolysis and fermentation (SHF), simultaneous saccharification and fermentation (SSF) and consolidated bioprocessing (CBP) [9-14]. SHF and SSF were carried out in the context of on-site cellulase production because this mode has many advantages such as enabling cost saving cost and tailor-made enzyme for a given feedstock [13-15]. CBP by an artificial microbial consortium composed of Trichoderma reesei Rut-C30 and the engineered S. cerevisiae strain was established for direct production of D-glucaric acid from lignocelluloses. Unlike the CBP by the same 
microbial consortium for bioethanol production where the ethanol production by $S$. cerevisiae was anaerobic but the cellulase production by $T$. reesei was aerobic [12], the CBP here was simpler because both the cellulase production by $T$. reesei and D-glucaric acid biosynthesis by $S$. cerevisiae were aerobic. The artificial microbial consortium for CBP was redesigned for higher efficiency, but the team of the two strains was found to be the best. CBP by the artificial microbial consortium achieved efficient D-glucaric acid production of lignocelluloses at a near gram per liter level. This work provides an example of Dglucaric acid production at record-breaking titer and presents the discovery that CBP of lignocellulose by the artificial microbial consortium is a desirable and promising approach for D-glucaric acid production.

\section{Methods}

\section{Plasmids and their construction}

The two heterologous genes, miox4 gene encoding MIOX4 enzyme in $A$. thaliana [16] and the udh gene encoding UDH in P. syringae [17], were codon-optimized for the expression in S. cerevisiae and synthesized by Sangon Biotech (Shanghai, China). Then these two genes were ligated to the plasmid of pY26-GPD-TEF, purchased from Miaoling Bioscience \& Technology Co., Ltd. (Wuhan, China), using Bg/ll/Notl and EcoRI/Xhol, to generate pY26-miox4-udh (Table S1). This recombinant vector carrying miox 4 and $u d h$ under the control of the promoters Pgpd and Ptef, respectively, were used for the subsequent transformation of $S$. cerevisiae.

pUG6 from Miaoling Bioscience \& Technology Co., Ltd. (Wuhan, China) with kanamycin resistance gene was used as the template for constructing the knock out cassette loxP-Kan-loxP by polymerase chain reaction (PCR) using the primers knock-OPI1F/R (Table S2). The plasmid pSH47 from Miaoling Bioscience \& Technology Co., Ltd. (Wuhan, China) provided Cre recombinase for the self-recombination of the knock out cassette.

The plasmid pCAMBIA1300 $[18,19]$ was used as the backbone for constructing the recombinant vector pCA-Pcbh1-ips-Tcbh1 (Table S1), where the gene ips (GenBank: L23520.1) encoding myo-inositol-1phosphate synthase from $S$. cerevisiae [2] was codon-optimized according to the codon preference of $T$. reesei and expressed heterologously under the control of the strong promoter Pcbh1 to improve the myoinositol production.

\section{Strains and media}

All strains used in this work, including the starting strains and the engineered strains, are listed in Table S1.

LB medium, containing $1 \mathrm{~g} / \mathrm{L}$ tryptone, $0.5 \mathrm{~g} / \mathrm{L}$ yeast extract, and $1 \mathrm{~g} / \mathrm{L} \mathrm{NaCl}$, was used to culture $E$. coli cells and Agrobacterium tumefaciens cells after being autoclaved at $121^{\circ} \mathrm{C}$ for $20 \mathrm{~min}$. YPD medium was used to culture $S$. cerevisiae cells, which had a following composition $(\mathrm{g} / \mathrm{L}): 10$ yeast extract, 20 peptone, 
and 20 glucose. When selective YPD medium was prepared, geneticin G418 was added to a specific concentration after being autoclaved and cooled down. Solid media were prepared by adding $2 \mathrm{~g} / \mathrm{L}$ agar before autoclave.

The seed medium for $T$. reesei strains was composed of $10 \mathrm{~g} / \mathrm{L}$ glucose, $1 \mathrm{~g} / \mathrm{L}$ peptone, $5 \mathrm{~mL}$ Mandels nutrient salts solution [20], $2.5 \mathrm{~mL}$ citrate buffer (1 mol/L), $0.05 \mathrm{~mL}$ Mandels trace elements solution [20], and $0.1 \mathrm{~g} / \mathrm{L}$ Tween 80 . The seed medium was autoclaved at $121^{\circ} \mathrm{C}$ for $20 \mathrm{~min}$. The fermentation medium for cellulase production by $T$. reesei comprised of $15 \mathrm{~g} / \mathrm{L}$ Avicel or $30 \mathrm{~g} / \mathrm{L}$ pretreated lignocellulose (dry biomass), $1 \mathrm{~g} / \mathrm{L}$ glucose, $6 \mathrm{~g} / \mathrm{L}\left(\mathrm{NH}_{4}\right)_{2} \mathrm{SO}_{4}, 2.0 \mathrm{~g} / \mathrm{L} \mathrm{KH}_{2} \mathrm{PO}_{4}, 0.3 \mathrm{~g} / \mathrm{L} \mathrm{CaCl}_{2}, 0.3 \mathrm{~g} / \mathrm{L} \mathrm{MgSO}{ }_{4}, 0.005 \mathrm{~g} / \mathrm{L}$ $\mathrm{FeSO}_{4}, 0.0016 \mathrm{~g} / \mathrm{L} \mathrm{MnSO}_{4}, 0.0014 \mathrm{~g} / \mathrm{L} \mathrm{ZnSO}_{4}$ and $0.0037 \mathrm{~g} / \mathrm{L} \mathrm{CoCl}_{2}$. The initial $\mathrm{pH}$ was adjusted to 4.8 with citrate buffer. This fermentation medium was autoclaved at $121^{\circ} \mathrm{C}$ for $30 \mathrm{~min}$.

All chemicals except lignocellulosic materials were purchased from Sinopharm Chemical Reagent Co. Ltd., Shanghai, China. The media for SSF and CBP were detailed in the Methods sections about them.

\section{Genetic engineering of $S$. cerevisiae}

The cassette loxP-Kan-loxP amplified from pUG6 by PCR using the primers knock-OPI1F/R (Table S2) was transformed into the competent cells of $S$. cerevisiae INVSc1 prepared with the Li-Ac method [21] to knock out opi1. The KanMX gene disruption cassette was cured by the homologous recombination between the loxP sites mediated by Cre recombinase expressed by the plasmid pSH47. pSH47 was lost as the host cells were cultivated continuously, resulting in the $S$. cerevisiae INVSc1 opi1 $\Delta$ strain which was used as the host for constructing the biosynthetic pathway for D-glucaric acid production.

There were two ways to express foreign genes, episomal or integrative expression in S. cerevisiae. For the episomal expression, the recombinant vector pY26-miox4-udh was directly transformed into the competent cells of S. cerevisiae INVSc1 opi1 . For the integrative expression, the expression cassettes were spliced by overlap PCR using delta1 and delta2 and integrated into Ty loci [3, 22]. First, delta1 and delta2 were amplified from $S$. cerevisiae genome using the primers delta1-F/delta1-R and delta2-F/delta2$\mathrm{R}$ (Table S2) respectively, and the miox 4 and $u d h$ expression cassettes were amplified from the plasmid pY26-miox4-udh using the primers MIXO4-F/R and UDH-F/R respectively. Second, the fragments d1-M-F and L-U-d2 were generated by the overlap PCR from delta1 and MIXO4 expression cassette using the primers delta1-F and FURA3-R and from UDH expression cassette and delta2 using the primers LURA$F /$ delta2-R, respectively. Finally, the whole fragment was gained from the overlap PCR from the fragments d1-M-F and L-U-d2 using the primers delta1-F and delta2-R, which was purified with Cyle-Pure Kit 200 (Omega Bio-tek, Georgia, USA) and then used for the transformation of the competent cells of $S$. cerevisiae INVSc1 opi14.

\section{Genetic engineering of $T$. reesei}


The recombinant plasmid pCA-Pcbh1-ips-Tcbh1 was transformed into T. reesei Rut-C30 by the method of A. tumefaciens mediated transformation (AMT) [23]. Then the potential T. reesei transformants were selected by the two rounds of screening, the first PDA (potato dextrose agar) plates added with hygromycin B and the second Avicel plates as described above $[18,19]$. The fast-growing $T$. reesei transformants selected by the two rounds of screening were tested in myo-inositol production and used in the artificial microbial consortium to increase myo-inositol availability.

\section{Fermentation for D-glucaric acid production by the engineered $S$. cerevisiae}

Fermentation was carried out in $250 \mathrm{~mL}$ Erlenmeyer flasks with a working volume $50 \mathrm{~mL}$ of YPD medium with or without $10.8 \mathrm{~g} / \mathrm{L}(60 \mathrm{mM})$ myo-inositol. Before inoculation into fermentation medium, $S$. cerevisiae strains were precultured in $5 \mathrm{~mL}$ YPD medium with $10.8 \mathrm{~g} / \mathrm{L}(60 \mathrm{mM})$ myo-inositol in $50 \mathrm{~mL}$ Erlenmeyer flasks at $30^{\circ} \mathrm{C}$ with a shaking of $250 \mathrm{rpm}$ to an optical density at $600 \mathrm{~nm}\left(\mathrm{OD}_{600}\right)$ of $\sim 5$. Then, the cells were collected and inoculated into fermentation medium with or without $10.8 \mathrm{~g} / \mathrm{L}(60 \mathrm{mM})$ myo-inositol to an $\mathrm{OD}_{600}$ of 0.1 . Fermentation was implemented at $30^{\circ} \mathrm{C}$ with a shaking of $250 \mathrm{rpm}$.

Fed-batch fermentation was conducted under the same conditions as the batch fermentation mentioned above, except that $5 \mathrm{~g} / \mathrm{L}$ glucose was supplemented twice during the fermentation process, $24 \mathrm{~h}$ and $48 \mathrm{~h}$ respectively.

\section{Separated hydrolysis and fermentation (SHF)}

In the scenario of SHF (Fig. 1), the cellulase was produced by T. reesei Rut-C30 from Avicel or steam exploded corn stover (SECS) $[14,24,25]$ was applied to the enzymatic hydrolysis of themselves, so called "on-site cellulase production" [13-15, 25]. T. reesei Rut-C30 was precultured in the seed medium for $36 \mathrm{~h}$ and then the seed collected and inoculated into the fermentation medium for cellulase production. The fermentation broth containing crude cellulase was directly used in the enzymatic hydrolysis of Avicel or SECS [14, 19], which was operated in $250 \mathrm{~mL}$ Erlenmeyer flasks with a working volume of $50 \mathrm{~mL} 2.5 \mathrm{~mL} 1$ $M$ citrate buffer solution (for final pH 4.8), $50 \mathrm{~g} / \mathrm{L}$ Avicel or $100 \mathrm{~g} / \mathrm{L} \mathrm{SECS} \mathrm{(dry} \mathrm{material),} 25 \mathrm{FPIU} / \mathrm{g}$ glucan the cellulase harvested after $5 \mathrm{~d}$ fermentation, and a supplementary amount of water to make up $50 \mathrm{~mL}$. The enzymatic hydrolysis was conducted at $50^{\circ} \mathrm{C}$ with a shaking of $140 \mathrm{rpm}$ for $48 \mathrm{~h}$. The resulted enzymatic hydrolysates containing fermentable sugars were used to prepare the fermentation medium supplemented with the same nutrients as YPD medium except glucose. The following operations were the same as those described in the section of fermentation for D-glucaric acid production by the engineered S. cerevisiae.

\section{Simultaneous saccharification and fermentation (SSF)}

In the scenario of SSF (Fig. 1), the cellulase prepared by the same method in SHF was used in the enzymatic prehydrolysis of Avicel or SECS for $12 \mathrm{~h}$. The prehydrolysis of SSF was performed in $250 \mathrm{~mL}$ Erlenmeyer flasks with $50 \mathrm{~mL} \mathrm{SSF}$ reaction mixture containing $50 \mathrm{~g} / \mathrm{L}$ Avicel or $100 \mathrm{~g} / \mathrm{L} \mathrm{SECS} \mathrm{(dry}$ material), $25 \mathrm{FPIU} / \mathrm{g}$ glucan the cellulase harvested after $5 \mathrm{~d}$ fermentation, $6 \mathrm{~g} / \mathrm{L}\left(\mathrm{NH}_{4}\right)_{2} \mathrm{SO}_{4}, 2.0 \mathrm{~g} / \mathrm{L}$ 
$\mathrm{KH}_{2} \mathrm{PO}_{4}, 0.3 \mathrm{~g} / \mathrm{L} \mathrm{MgSO}{ }_{4} \cdot 7 \mathrm{H}_{2} \mathrm{O}, 0.3 \mathrm{~g} / \mathrm{L} \mathrm{CaCl}_{2} \cdot 2 \mathrm{H}_{2} \mathrm{O}, 0.1 \mathrm{~g} / \mathrm{L}$ Tween $80,10 \mathrm{~g} / \mathrm{L}$ peptone, $5 \mathrm{~g} / \mathrm{L}$ yeast extract, and a supplementary amount of water to make up $50 \mathrm{~mL}$. Before adding cellulase, the initial $\mathrm{pH}$ was adjusted to 4.8 with citrate buffer and the reaction mixture was autoclaved at $121^{\circ} \mathrm{C}$ for $30 \mathrm{~min}$. After cellulase addition, the reaction mixture was incubated at $50^{\circ} \mathrm{C}$ with a shaking of $140 \mathrm{rpm}$ for $12 \mathrm{~h}$. Then the temperature was decreased to $33^{\circ} \mathrm{C}$ (or specified in the main text when studying its effect on SSF) and the shaking was increased to $250 \mathrm{rpm}$. The precultured $S$. cerevisiae with an $\mathrm{OD}_{600}$ of $\sim 5$ was inoculated into the reaction mixture for SSF.

\section{Consolidated Bioprocessing (CBP)}

The medium for CBP had a following composition: $15 \mathrm{~g} / \mathrm{L}$ (or specified in the main text when investigating its effect on CBP) Avicel or SECS (dry material), $1 \mathrm{~g} / \mathrm{L}$ peptone, $1 \mathrm{~g} / \mathrm{L}$ yeast extract, $10 \%(\mathrm{v} / \mathrm{v})$ Mandels nutrient salts solution [20], $0.1 \%(\mathrm{v} / \mathrm{v})$ trace elements solution [20], $5 \%(\mathrm{v} / \mathrm{v})$ citrate buffer (1 $\mathrm{mol} / \mathrm{L}$ ), $0.1 \mathrm{~g} / \mathrm{L}$ Tween 80 . CBP medium was autoclaved at $121^{\circ} \mathrm{C}$ for $30 \mathrm{~min}$. T. reesei was precultured in the seed medium at $30^{\circ} \mathrm{C}$ for $36 \mathrm{~h}$ and $S$. cerevisiae was precultured in YPD medium at $30^{\circ} \mathrm{C}$ till an $\mathrm{OD}_{600}$ of $\sim 5$. Then $T$. reesei was inoculated into CBP medium and the inoculation of $S$. cerevisiae was implemented immediately or delayed (or specified in the main text when studying its effect on CBP). The inoculum ratio of $T$. reesei to $S$. cerevisiae was 1:1 (or specified in the main text when studying its effect on CBP). If other strain or species was inoculated, the detailed information would be specified in the main text. The total inocula were $10 \%(\mathrm{v} / \mathrm{v})$ of the fermentation medium. CBP was carried out in $250 \mathrm{~mL}$ Erlenmeyer flasks with $50 \mathrm{~mL}$ medium at $30^{\circ} \mathrm{C}$ with a shaking of $180 \mathrm{rpm}$. A. niger was precultured for 48 $\mathrm{h}$ by the same method as $T$. reesei if needed.

\section{Analytical methods}

Filter paper activity (FPA) of cellulase, representing the total enzymatic activity, was assayed by the method standardized by the International Union of Pure and Applied Chemistry (IUPAC) [26], which quantifies the total amount of the reducing sugars produced from $50 \mathrm{mg}$ Whatman No.1 filter paper $(1 \times 6$ $\mathrm{cm}$ strip) by cellulase within $60 \mathrm{~min}$. One International Unit of FPA (FPIU) was defined as the amount of cellulase needed for producing $1 \mu \mathrm{mol}$ reducing sugars in $1 \mathrm{~min}$.

$\beta$-Glucosidase activity (BGA) was determined using the standard method [26] with the tiny modification, i.e. the substrate $\rho N P G$ ( $\rho$-nitrophenyl- $\beta$-d-1,4-glucopiranoside) (Sigma-Aldrich, St. Louis, MO, USA). The amount of $\rho$-nitrophenol produced from $\rho N P G$ by $\beta$-glucosidase within 10 min was assayed using spectrophotometer at a wavelength of $400 \mathrm{~nm}$. One International Unit of BGA (IU) was defined as the amount of $\beta$-glucosidase required for producing $1 \mu \mathrm{mol}$ of $\rho$-nitrophenol from $\rho N P G$ in $1 \mathrm{~min}$.

Cellobiohydrolase activity (BGA) was assayed according to the method modified from FPA measurement method [26]. Microcrystalline cellulose PH101 purchased from Sigma-Aldrich (St. Louis, MO, USA) in the form of $1 \%(\mathrm{w} / \mathrm{v})$ suspension was used as the substrate for the reaction with the duration of $30 \mathrm{~min}$. One Unit $(1 \mathrm{U})$ of CBA was defined as the amount of enzyme required for producing $1 \mathrm{mg}$ reducing sugars in 1 h. 
High performance liquid chromatography (HPLC) was adopted to analyze and quantify D-glucaric acid, myo-inositol and sugars, where Shimadzu Prominence LC-20A system equipped with Bio-Rad Aminex HPX-87H (300 mm×7.8 mm) column was used. When D-glucaric acid was determined, an ultraviolet (UV) detector was employed to detect the eluate. While myo-inositol and sugars were determined, a refractive index (RI) detector was employed. Sulfuric acid ( $5 \mathrm{mmol} / \mathrm{L})$ was used as the mobile phase and set at a flow rate of $0.6 \mathrm{~mL} / \mathrm{min}$. The column temperature was maintained at $50^{\circ} \mathrm{C}$. Sample loading for each injection was $10 \mu \mathrm{L}$.

Liquid Chromatography-Mass Spectrometry (LC-MS) was used to characterize and identify D-glucaric acid, where Shimadzu L-30A and AB Sciex Triple TOF 5600 were employed. The column was Shimadzu Shim-pack XR-ODS 100L $\times 2.0$. The mobile phases, set at a flow rate of $0.15 \mathrm{~mL} / \mathrm{min}$, were the aqueous solution containing $1 \mathrm{mmol} / \mathrm{L}$ ammonium formate and 1\%(v/v) formic acid (Mobile phase $\mathrm{A}$ ) and the acetonitrile solution containing $1 \mathrm{mmol} / \mathrm{L}$ ammonium formate (Mobile phase $\mathrm{B}$ ), respectively. Mobile phase $B, B$ for short and the same below, was used as the eluent and the elution procedure was as follows: 0-12 min 30\% B, 12-30 min 30\%-65\% B, 30-31 min 65\%-95\% B, 31-35 min 95\% B, 35-36 min $95 \%-30 \%$ B, 36-40 min 30\% B. Sample loading for each injection was $5 \mu \mathrm{L}$. Negative electrospray ionization mode was chosen to ionize samples. The flow rate of atomizing gas $\left(\mathrm{N}_{2}\right)$ was $1.5 \mathrm{~L} / \mathrm{min}$. The temperature for $\mathrm{CDL}$ and $\mathrm{HB}$ was $200^{\circ} \mathrm{C}$. Scanning scope $(\mathrm{m} / \mathrm{z})$ ranged from 150 to 300 .

\section{Results}

\section{Construction of D-glucaric acid-producing S. cerevisiae}

It was reported that the availability of myo-inositol was the limiting step for D-glucaric acid in $S$. cerevisiae $[3,5]$, whose synthesis is negatively regulated by opi $1[8,27]$. In order to relieve the negative regulation of opi1 on myo-inositol synthesis, therefore, opi1 was knocked out to generate $S$. cerevisiae strain INVSc1 opi $1 \Delta[3,5]$, which was subsequently used as the starting strain for the construction of Dglucaric acid-producing S. cerevisiae, as detailed in Methods. The biosynthetic pathway of D-glucaric acid in the engineered S. cerevisiae is illustrated in Fig. 2A. It was also found here that the deletion of opi1 elevated D-glucaric acid production (data not shown), which was in line with the peer's report [3]. Two Dglucaric acid-producing strains derived from S. cerevisiae strain INVSc1 opi1 $\Delta$, S. cerevisiae LGA-1 with the integrative expression of foreign genes and $S$. cerevisiae LGA-C with the episomal expression, were chosen for subsequent fermentation experiments. The products of $S$. cerevisiae LGA-1 and LGA-C were characterized by LC-MS to verify their capability of producing D-glucaric acid and the results are confirmative, as shown in Fig. S1.

\section{Fed-batch fermentation with or without myo-inositol}

These two strains, S. cerevisiae LGA-1 and S. cerevisiae LGA-C, were compared in the fermentations on YPD medium without and with $10.8 \mathrm{~g} / \mathrm{L}(60 \mathrm{mM})$ myo-inositol $[3,5]$. The time courses of the fermentations are shown in Fig. 2B. It was found that the fermentation processes supplemented with 
myo-inositol had higher D-glucaric acid titer than those using glucose as sole carbon source. This observation is the same as the previous reports [3,5]. LGA-1 performed similarly to LGA-C when using glucose as sole carbon source, in contrast to their performances in the fermentation supplemented with myo-inositol where LGA-C outperformed LGA-1. After $7 \mathrm{~d}$ fermentation, LGA-1 produced $9.53 \pm 0.46 \mathrm{~g} / \mathrm{L}$ and LGA-C produced $11.21 \pm 0.63 \mathrm{~g} / \mathrm{L}$ D-glucaric acid from $30 \mathrm{~g} / \mathrm{L}$ glucose and $10.8 \mathrm{~g} / \mathrm{L}$ myo-inositol in the mode of fed-batch fermentation, respectively.

Production of D-glucaric acid from myo-inositol (marked with yellow arrows in Fig. 2A), however, is not a desirable approach because the latter is also an industrially value-added chemical $[28,29]$. Moreover, so far there has not been any reports pertaining to D-glucaric acid production from lignocellulose, though it was considered as one of the top value-added chemicals from biomass [1]. Therefore, production of Dglucaric acid from lignocellulose in the context of biorefinery, the same as production of lignocellulosic biofuels such as lignocellulosic ethanol $[9,10]$, should be treated as an important attempt to seek cheaper biomanufacturing of D-glucaric acid. Hence, LGA-C and LGA-1 were applied to the D-glucaric acid production from Avicel and pretreated corn stover via SHF, SSF and CBP, typical bioprocesses in biorefinery.

\section{Separated hydrolysis and fermentation (SHF)}

The time courses of cellulase production from Avicel and SECS are presented in Fig. 3A. Avicel induced slightly higher FPA than SECS in the cellulase production by T. reesei Rut-C30. On Day 5, the FPAs induced by Avicel and SECS were $2.87 \pm 0.29$ and $2.45 \pm 0.36 \mathrm{FPIU} / \mathrm{mL}$, respectively. Then FPAs decreased because $T$. reesei Rut-C30 entered decline phase, which is in line with the previous work $[18,30]$. Thus, the cellulases harvested on Day 5 were applied to the enzymatic hydrolysis of themselves in the context of on-site cellulase production [13-15], i.e. the cellulase induced by Avicel used for the enzymatic hydrolysis of Avicel, because this is advantageous over the cellulases induced by other substrates or commercial cellulases $[15,31]$. The results of the enzymatic saccharification of Avicel and SECS are shown in Fig. 3B. Enzymatic hydrolysis of SECS was found to have higher yield than that of Avicel. The former produced $39.73 \pm 0.95 \mathrm{~g} / \mathrm{L}$ glucose from $100 \mathrm{~g} / \mathrm{L}$ SECS containing $53.2 \mathrm{~g} / \mathrm{L}$ glucan and the latter produced $28.31 \pm 1.17 \mathrm{~g} / \mathrm{L}$ glucose from $50 \mathrm{~g} / \mathrm{L}$ Avicel. The resulted hydrolysates containing fermentable sugars were subsequently fermented by the engineered $S$. cerevisiae strains, LGA1 and LGA-C, for D-glucaric acid production. As shown in Fig. 3C, the time courses were in the similar pattern. Though the enzymatic hydrolysate of SECS fermented by LGA-1 led to the highest D-glucaric acid titer of $4.92 \pm 0.24 \mathrm{~g} / \mathrm{L}$ after $7 \mathrm{~d}$ fermentation, the differences were not so distinguishable, let alone the enzymatic hydrolysate of Avicel had lower concentration of glucose.

Overall, LGA-1 produced $3.74 \pm 0.31 \mathrm{~g} / \mathrm{L}$ D-glucaric acid and LGA-C produced $3.93 \pm 0.38 \mathrm{~g} / \mathrm{L}$ D-glucaric acid from $50 \mathrm{~g} / \mathrm{L}$ Avicel. LGA-1 produced $4.92 \pm 0.24 \mathrm{~g} / \mathrm{L}$ D-glucaric acid and LGA-C produced $4.63 \pm 0.52$ $\mathrm{g} / \mathrm{L}$ D-glucaric acid from $100 \mathrm{~g} / \mathrm{L}$ SECS.

\section{Simultaneous saccharification and fermentation (SSF)}


Three temperatures, 30,33 and $36^{\circ} \mathrm{C}$, were tested here to study the effect on SSF and the results are shown in Fig. S2, Fig. 4 and Fig. S3, respectively. It was found that $33^{\circ} \mathrm{C}$ was the most suitable temperature for SSF, leading to the highest concentrations of D-glucaric acid. This is the same as SSF for bioethanol production where $33^{\circ} \mathrm{C}$ was also the most suitable temperature [11].

The time courses of SSF for D-glucaric acid production from Avicel and SECS are shown Fig. 4A and B, respectively, which displayed similar pattern that glucose dropped rapidly after LGA-1 or LGA-C being inoculated. After $48 \mathrm{~h}$, glucose reduced to an extremely low level close to zero. The titers of D-glucaric acid increased quickly after $12 \mathrm{~h}$, the time point of $S$. cerevisiae inoculation, and reached the plateau after $72 \mathrm{~h}$. LGA-1 and LGA-C had reverse results in the SSF for producing D-glucaric acid. The former produced the higher titer of D-glucaric acid than the latter from Avicel but the former produced lower titer from SECS. However, the differences were not so enormous.

After $7 \mathrm{~d}$, LGA-1 produced $1.22 \pm 0.28 \mathrm{~g} / \mathrm{L}$ D-glucaric acid and LGA-C produced $1.43 \pm 0.20 \mathrm{~g} / \mathrm{L} \mathrm{D}$-glucaric acid from $50 \mathrm{~g} / \mathrm{L}$ Avicel. LGA-1 produced $1.14 \pm 0.19 \mathrm{~g} / \mathrm{L}$ D-glucaric acid and LGA-C produced $1.02 \pm 0.13$ $\mathrm{g} / \mathrm{L}$ D-glucaric acid from $100 \mathrm{~g} / \mathrm{L}$ SECS.

\section{Consolidated bioprocessing (CBP)}

$\mathrm{CBP}$ is a desirable approach for D-glucaric acid production from lignocellulose because both cellulase production by $T$. reesei and D-glucaric acid production by $S$. cerevisiae are aerobic, unlike CBP for bioethanol production where an anaerobic condition should be created for $S$. cerevisiae [12]. Here the operation of CBP was much easier. CBP for D-glucaric acid production from lignocellulose by $T$. reesei and S. cerevisiae is illustrated in Fig. 5A. The microbial consortium composed of T. reesei Rut-C30 and the engineered $S$. cerevisiae could achieve the bioconversion of lignocellulose to D-glucaric acid in one step.

The time courses of CBPs from Avicel and SECS are shown in Fig. 5B and C, respectively. It was found that the microbial consortium of $T$. reesei Rut-C30 and S. cerevisiae LGA-1 produced $0.54 \pm 0.12 \mathrm{~g} / \mathrm{L} \mathrm{D}$ glucaric acid from $15 \mathrm{~g} / \mathrm{L}$ Avicel, and $0.45 \pm 0.06 \mathrm{~g} / \mathrm{L}$ D-glucaric acid from $15 \mathrm{~g} / \mathrm{L}$ SECS after $7 \mathrm{~d}$ fermentation. Somewhat lower concentrations of D-glucaric acid were produced when $S$. cerevisiae LGA-C was used. Thus, S. cerevisiae LGA-1 was more desirable in the CBP for D-glucaric acid production. Increase in substrate loading did not lead to higher concentrations of D-glucaric acid, as shown in Fig. S4. The optimal substrate loading in CBP for D-glucaric acid here was lower than that in CBP for bioethanol, $17.5 \mathrm{~g} / \mathrm{L}$ [12]. This result implicates the low efficiency of the microbial consortium comprising of $T$. reesei Rut-C30 and S. cerevisiae LGA-1 in CBP of lignocellulose to D-glucaric acid.

Further work was thus carried out to improve the efficiency. The effect of the ratio of $T$. reesei to $S$. cerevisiae on CBP was investigated and the results are shown in Fig. S5. Among the inoculum ratios of $T$. reesei to $S$. cerevisiae, 1:1, 1:3, 1:5, 5:1 and 3:1, 1:1 was found to be the best for CBP. Changing the ratio caused lower D-glucaric acid titer, proven incapable of improving the efficiency. The effect of the delay time of S. cerevisiae inoculation on CBP was also studied and the results are presented in Fig. S6. Among 
the delay times of $S$. cerevisiae inoculation, 0,24 and 48 h, 0 (i.e. T. reesei and S. cerevisiae were inoculated simultaneously) was found to be the most suitable.

In the light of the mixed culture of $T$. reesei and $A$. niger for enhanced cellulase production [30, 32], $A$. niger was introduced into the microbial consortium to improve the efficiency of lignocellulose degradation. The ratio of $T$. reesei to $A$. niger to $S$. cerevisiae was 5:1:5 and the ratio of the total inocula to fermentation medium was $10 \%(\mathrm{v} / \mathrm{v})$. The results are presented in Fig. $6 \mathrm{~A}, \mathrm{D}, \mathrm{E}, \mathrm{F}$ and $\mathrm{G}$, and it was found that the microbial consortium composed of $T$. reesei, $A$. niger and $S$. cerevisiae produced lower concentrations of D-glucaric acid than the microbial consortium consisted of $T$. reesei and $A$. niger, though the former produced higher FPA. This suggests that the microbial consortium of T. reesei, A. niger and $S$. cerevisiae was not suitable for CBP of lignocellulose for D-glucaric acid production.

Gupta et al [5]. found that myo-inositol availability was the rate-limiting step in the engineered $S$. cerevisiae expressing miox4 gene from $A$. thaliana. The same strategy was used here. Thus, we adopted the following strategies to increase myo-inositol availability. We engineered $T$. reesei Rut-C30 to enable it accumulating extracellular myo-inositol by expressing inositol-3-phosphate synthase (ips, GenBank: L23520.1) and inositol monophosphatase (imp, GenBank: CP029160.1) from S. cerevisiae. But this was proven unsuccessful because engineering $T$. reesei to produce myo-inositol made it no longer potent in cellulase production, let alone the engineered $T$. reesei produced a negligible concentration of myoinositol (Fig. S7). The results in Fig. 6B, D, E, F and G indicate that this strategy was not feasible to improve D-glucaric acid production by CBP of lignocellulose.

Then, we turned eyes to the engineered $S$. cerevisiae with opi1 gene being knocked out, whose myoinositol accumulation was improved (Fig. S8), i.e. the parental strain of LGA-1 and LGA-C. S. cerevisiae $\triangle o p i 1$ was added to the microbial consortium to try to improve the efficiency of CBP for D-glucaric acid production. The inoculum ratio of $T$. reesei to $S$. cerevisiae $\triangle$ opi1 to $S$. cerevisiae LGA-1 was 2:1:1 and the total volume of the inocula was $10 \%(\mathrm{v} / \mathrm{v})$ of fermentation medium. It was found in Fig. $6 \mathrm{C}, \mathrm{D}, \mathrm{E}, \mathrm{F}$ and $\mathrm{G}$ that this strategy was inferior to that strategy described in Fig. 5A.

Overall, the microbial consortium of $T$. reesei Rut-C30 and S. cerevisiae LGA-1 was the most efficient CBP for D-glucaric acid production from lignocellulose. Addition of the third strain or metabolic engineering of T. reesei for enhanced myo-inositol availability was proven unsuccessful in improving the efficiency of CBP.

\section{Comparison of different biorefinery processes for D-glucaric acid production}

The parameters of different biorefinery processes for D-glucaric production from Avicel and SECS, including SHF, SSF and CBP, are listed in Table 1 and 2, respectively. The fermentation processes from pure glucose with or without myo-inositol supplementation were used as the references for comparison. It is obvious that fermentation on glucose supplemented with myo-inositol had the highest D-glucaric acid titer. Next in line is the D-glucaric acid production from glucose or from the hydrolysates of SECS and Avicel containing glucose. SHF had a D-glucaric acid titer close to that from pure glucose without myo- 
inositol supplementation. SSF had lower D-glucaric acid titers from Avicel and SECS than those of SHF, and much lower yields than those of SHF. This suggests that SSF was not a desirable approach for Dglucaric acid production from lignocellulose due to its low efficiency.

Though CBP had relatively low D-glucaric acid titer, it had considerably high yields from Avicel and SECS. This is because low substrate loading was used. The yields of CBPs were $0.0360 \mathrm{~g}$ D-glucaric acid/g Avicel and $0.0300 \mathrm{~g} \mathrm{D-glucaric} \mathrm{acid/g} \mathrm{SECS,} \mathrm{higher} \mathrm{than} \mathrm{those} \mathrm{of} \mathrm{SSF} \mathrm{but} \mathrm{a} \mathrm{little} \mathrm{lower} \mathrm{than} \mathrm{those}$ of SHF (0.0593 g D-glucaric acid/g Avicel and $0.0377 \mathrm{~g} \mathrm{D-glucaric} \mathrm{acid/g} \mathrm{SECS).} \mathrm{Moreover,} \mathrm{CBP} \mathrm{has} \mathrm{the}$ least single unit operations and the greatest potential of cost reduction [10, 12,33]. In addition, both the cellulase production by $T$. reesei Rut-C30 and D-glucaric acid production by $S$. cerevisiae LGA-1 during CBP of lignocellulose by the microbial consortium are aerobic. This reduces the difficulty and complexity of constructing and operating the microbial consortium [12]. Therefore, this is highly promising approach for D-glucaric production from lignocellulose.

\section{Discussion}

The strategy of constructing S. cerevisiae strain for D-glucaric acid production at high titer was applied to a different yeast strain S. cerevisiae INVSc1 in this work [3]. Two engineered strains with high titers of Dglucaric acid, LGA-1 and LGA-C, were obtained. LGA-C with the episomal expression of miox4 and udh had higher concentration of D-glucaric acid than LGA-1 with the integrated expression when supplementing myo-inositol (Fig. 2B). But no obvious difference observed when using glucose as sole carbon source. This may be because episomal expression was more efficient in transforming myoinositol into D-glucaric acid than integrated expression when abundant myo-inositol was available, or the recombinant plasmid with high copy number was led to more MIOX4 and UDH than the integration into Ty loci. In practice, both LGA-1 and LGA-C acted as whole cell catalysts when the fermentation supplemented with myo-inositol. In the fed-batch fermentation supplemented with myo-inositol, both LGA-1 and LGA-C produced the record-breaking titer of D-glucaric acid, $9.53 \pm 0.46 \mathrm{~g} / \mathrm{L}$ and LGA-C produced $11.21 \pm 0.63 \mathrm{~g} / \mathrm{L} \mathrm{D}$-glucaric acid, much higher than the previous reports on $S$. cerevisiae $[3,5]$ and $E$. coli $[2,4,7,34]$. It is noteworthy that higher D-glucaric acid was produced, though we adopted the same strategy as Chen et al [3]. This is probably because different $S$. cerevisiae strain was used. Different strain means different physiological statuses, different efficiencies of foreign gene expressions, different myo-inositol availabilities, etc.

The high titer of D-glucaric acid produced by $S$. cerevisiae LGA-C demonstrates the great potential of $S$. cerevisiae INVSc1 in D-glucaric acid production, although episomal expression is problematic because of its genetic instability [3]. The higher titer of D-glucaric acid produced from glucose and myo-inositol than that from glucose suggests that myo-inositol availability is still the rate-liming step for D-glucaric acid production in the engineered $S$. cerevisiae. Furthermore, myo-inositol is a valuable chemical in industry $[28,29]$. The production of D-glucaric acid from myo-inositol was not so economically competitive as the direct production from glucose. In order to enhance the D-glucaric acid production 
from glucose, therefore, further work should be done to improve the biosynthetic pathway efficiency of Dglucaric acid in S. cerevisiae.

As one of the top value-added chemicals from biomass [1], there had been no reports on D-glucaric acid production from lignocellulose, the most abundant renewable on earth. Three biorefinery processes, SHF, SSF and CBP (Fig. 1), were conducted using the engineered S. cerevisiae strains, LGA-1 and LGA-C. Two substrates were used in these biorefinery processes, Avicel and SECS. The former is pure cellulose that is often used as model substrate, and the latter is corn stover that is abundant in China but always improperly treated, causing serious air pollution [31]. The results of SHF (Fig. 3) shows that Avicel induced more cellulase but had lower glucose concentrations. Overall, both D-glucaric acid titer and yield of SECS were comparable to those of Avicel (Table 1 and Table 2). Moreover, the results of SHF were close to fermentation from pure glucose. These indicate that corn stover a suitable feedstock for Dglucaric acid production and the engineered $S$. cerevisiae could be applied to the biorefinery of lignocellulose for D-glucaric production.

SSF was proven not so successful here, leading to the lowest D-glucaric acid titer and yields from Avicel and SECS (Table 1 and 2). This is because SSF is not authentic, which encompassed two phases, enzymatic prehydrolysis and SSF. Only the former phase had the highest hydrolysis efficiency. After entering SSF, temperature was decreased to the appropriate range of $S$. cerevisiae in which the enzymatic hydrolysis rate was reduced. If SSF temperature was increased for improved enzymatic hydrolysis, the fermentation by S. cerevisiae was weakened (Fig. 4, Fig. S2 and Fig. S3). The hurdle of SSF is the gap between the optimal temperature for enzymatic hydrolysis by $T$. reesei cellulase and that for fermentation by $S$. cerevisiae. This is the same as SSF for bioethanol production [11].

Enlightened by the previous research on CBP for bioethanol production [12], we constructed the same microbial consortium of $T$. reesei and $S$. cerevisiae and applied it to CBP of lignocellulose for D-glucaric acid production. The results (Fig. 5, Table 1 and 2) are promising because it had relatively high yields, demonstrating its efficiency. However, there is still room to increase the efficiency of CBP by $T$. reesei and $S$. cerevisiae. The ratio of $T$. reeseito $S$. cerevisiae was investigated and 1:1 was the most suitable one (Fig. S5). This makes sense because decreasing the ratio weakened the lignocellulose degrading capability and increasing the ratio attenuated the fermentation by the engineered $S$. cerevisiae for $D$ glucaric acid production. Then we delayed the inoculation of $S$. cerevisiae, resulting in reduced D-glucaric acid production. Simultaneous inoculations of $T$. reesei and $S$. cerevisiae were the most suitable. This is because delaying $S$. cerevisiae inoculation let $T$. reesei consumed more substrates and less was left for $D-$ glucaric acid production.

The cellulase of $T$. reesei Rut-C30 is deficient in $\beta$-glucosidase and the previous researches proved that the mixed culture of $T$. reesei and $A$. niger could improve the cellulase production and the cellulose deconstruction capability [30, 32, 35]. Here we introduced $A$. niger into the microbial consortium and the cellulase production during CBP was enhanced (Fig. 6). On contrary, D-glucaric acid concentration lowered. It is plausible that $A$. niger got more but contributed less in the team of the three members, 
therefore impairing the efficiency of CBP. The same situation happened when we introduced S. cerevisiae opi1 $\Delta$ into the microbial consortium to increase myo-inositol availability (Fig. 6 and Fig. S8). Then we engineered $T$. reesei Rut-C30 to make it able to provide myo-inositol during CBP. But once $T$. reesei was engineered, its cellulase production capability was crippled (Fig. S7). This made the microbial consortium weaker in lignocellulose degradation, negatively affecting the efficiency of CBP for D-glucaric acid production from lignocellulose (Fig. 6). The failure of metabolic engineering of $T$. reesei here discourages the further efforts to metabolically engineer $T$. reesei for direct production of D-glucaric acid from lignocellulose. Even if successful, it is nearly impossible to obtain a super strain simultaneously good at cellulase production and D-glucaric acid production. Microbial cell factory would be faced with serious metabolic dilemma when doing the two things at the same time [36]. Thus, the artificial microbial consortium of T. reesei Rut-C30 (cellulase specialist) and S. cerevisiae LGA-1 (D-glucaric acid specialist) was an excellent team with "chemistry" in CBP.

In the long run, direction production of D-glucaric acid from lignocellulose is advantageous over that from glucose, not to mention that from myo-inositol. Due to the highest integration of single unit operations, the utmost simplicity of bioprocess control, the cheapest substrate, and CBP is promising in the production of D-glucaric acid from lignocellulose. The relatively high D-glucaric acid titer and yield from this work proves that CBP of lignocellulose by the artificial microbial consortium of $T$. reesei and $S$. cerevisiae deserves extensive and in-depth research. The priority of the to-do list is to improve the efficiency of the CBP by the microbial consortium, still low at current stage. We are engineering $T$. reesei to enhance enzymatic hydrolysis and $S$. cerevisiae to improve biosynthesis of D-glucaric acid. The ultimate aim is to realize the distributive and collaborative push-and-pull strategy for promoting the CBP by the microbial consortium of $T$. reesei and S. cerevisiae for D-glucaric acid production from lignocellulose.

\section{Conclusions}

The biosynthetic pathway of D-glucaric acid production was constructed in S. cerevisiae INVSc1 whose opi1 was knocked out by expressing miox 4 from $A$. thaliana and udh from $P$. syringae, successfully obtaining two high titer D-glucaric acid producing strains, LGA-1 and LGA-C. Both of them produced record breaking titers of D-glucaric acid, indicating that $S$. cerevisiae INVSc1 was an excellent host. However, these high D-glucaric titers were facilitated by myo-inositol supplementation, which is not so preferable as using lignocellulose. SHF, SSF and CBP were studied here and it was found that CBP by an artificial microbial consortium of $T$. reesei Rut-C30 and the engineered $S$. cerevisiae was a promising approach with relatively high titer and yield. The microbial consortium was redesigned for higher efficiency, but the two members, $T$. reesei Rut-C30 and S. cerevisiae LGA-1, were proved to be the best teammates for CBP. Further work should be done to improve the efficiency of this microbial consortium for D-glucaric acid production from lignocellulose.

\section{Abbreviations}


AMT: Agrobacterium tumefaciens mediated transformation; BGA: beta-glucosidase activity; CBP: consolidated bioprocessing; FPA: filter paper activity; HPLC: high performance liquid chromatography; Ino1: myo-inositol-1-phosphate synthase; LC-MS: liquid chromatography-mass spectrometry; MIOX: myoinositol oxygenase; PDA: potato dextrose agar; RI: refractive index; SECS: steam-exploded corn stover; SHF: Separated hydrolysis and fermentation; SSF: Simultaneous saccharification and fermentation; UDH: urinate dehydrogenase; UV: ultraviolet.

\section{Declarations}

\section{Ethics approval and consent to participate}

Not applicable.

\section{Consent for publication}

Not applicable.

\section{Conflicts of interest}

There are no conflicts to declare.

\section{Funding}

This work was supported by National Natural Science Foundation of China (NSFC No. 22007079), the General Grant for Young Scholar (2018JQ2022) and the second-class General Postdoctoral Grant (2017BSHEDZZ100) from Shaanxi Province, the Special Funding and first-class General Financial Grants from the China Postdoctoral Science Foundation (2018T111102 and 2016M600815) and the Start-up Fund for Talent Introduction (Z111021602) from Northwest A\&F University.

\section{Authors' contributions}

$\mathrm{CL}$ and HF conceived the ideas and designed the project. $C L, X F L, X L$ and $S L$ performed the experiments.

$\mathrm{CL}$ and HF analyzed the data. HF wrote the manuscript. All authors have read and approved the final manuscript.

\section{Acknowledgements}

Not applicable.

\section{Availability of data and material}

Not applicable.

\section{Author details}


${ }^{a}$ College of Life Sciences, Northwest A\&F University, 22 Xinong Road, Yangling 712100, Shaanxi, China.

${ }^{b}$ Biomass Energy Center for Arid and Semi-arid Lands, Northwest A\&F University, 22 Xinong Road, Yangling 712100, Shaanxi, China.

\section{References}

1. Werpy T, Petersen G: Top value added chemicals from biomass, volume 1: results of screening for potential candidates from sugars and synthesis gas. US Department of Energy, Washington, DC 2004.

2. Moon TS, Yoon SH, Lanza AM, Roy-Mayhew JD, Prather KL: Production of glucaric acid from a synthetic pathway in recombinant Escherichia coli. Applied and Environmental Microbiology 2009, 75:589-595.

3. Chen N, Wang J, Zhao Y, Deng Y: Metabolic engineering of Saccharomyces cerevisiae for efficient production of glucaric acid at high titer. Microbial Cell Factories 2018, 17:67.

4. Shiue E, Prather KL: Improving D-glucaric acid production from myo-inositol in E. coli by increasing MIOX stability and myo-inositol transport. Metabolic Engineering 2014, 22:22-31.

5. Gupta A, Hicks MA, Manchester SP, Prather KL: Porting the synthetic D-glucaric acid pathway from Escherichia coli to Saccharomyces cerevisiae. Biotechnology Journal 2016, 11:1201-1208.

6. Smith TN, Hash K, Davey CL, Mills H, Williams H, Kiely DE: Modifications in the nitric acid oxidation of D-glucose. Carbohydrate Research 2012, 350:6-13.

7. Moon TS, Dueber JE, Shiue E, Prather KL: Use of modular, synthetic scaffolds for improved production of glucaric acid in engineered E. coli. Metabolic Engineering 2010, 12:298-305.

8. Ye C, Bandara WM, Greenberg ML: Regulation of inositol metabolism is fine-tuned by inositol pyrophosphates in Saccharomyces cerevisiae. The Journal of Biological Chemistry 2013, 288:2489824908.

9. Paulova L, Patakova P, Branska B, Rychtera M, Melzoch K: Lignocellulosic ethanol: Technology design and its impact on process efficiency. Biotechnology Advances 2015, 33:1091-1107.

10. Chung D, Cha M, Guss AM, Westpheling J: Direct conversion of plant biomass to ethanol by engineered Caldicellulosiruptor bescii. Proceedings of the National Academy of Sciences of the United States of America 2014, 111:8931-8936.

11. Zhao J, Xia L: Simultaneous saccharification and fermentation of alkaline-pretreated corn stover to ethanol using a recombinant yeast strain. Fuel Processing Technology 2009, 90:1193-1197.

12. Brethauer S, Studer MH: Consolidated bioprocessing of lignocellulose by a microbial consortium. Energy \& Environmental Science 2014, 7:1446.

13. Zhao C, Zou Z, Li J, Jia H, Liesche J, Chen S, Fang H: Efficient bioethanol production from sodium hydroxide pretreated corn stover and rice straw in the context of on-site cellulase production. Renewable Energy 2018, 118:14-24. 
14. Zhao C, Zou Z, Li J, Jia H, Liesche J, Fang H, Chen S: A novel and efficient bioprocess from steam exploded corn stover to ethanol in the context of on-site cellulase production. Energy 2017, 123:499510.

15. Culbertson A, Jin M, da Costa Sousa L, Dale BE, Balan V: In-house cellulase production from AFEX ${ }^{\mathbf{m}}$ pretreated corn stover using Trichoderma reesei RUT C-30. RSC Advances 2013, 3:25960.

16. Lorence A, Chevone BI, Mendes P, Nessler CL: myo-inositol oxygenase offers a possible entry point into plant ascorbate biosynthesis. Plant Physiology 2004, 134:1200-1205.

17. Yoon SH, Moon TS, Iranpour P, Lanza AM, Prather KJ: Cloning and characterization of uronate dehydrogenases from two pseudomonads and Agrobacterium tumefaciens strain C58. Journal of Bacteriology 2009, 191:1565-1573.

18. Fang $\mathrm{H}$, Xia L: High activity cellulase production by recombinant Trichoderma reesei ZU-02 with the enhanced cellobiohydrolase production. Bioresource Technology 2013, 144:693-697.

19. Fang H, Zhao R, Li C, Zhao C: Simultaneous enhancement of the beta-exo synergism and exo-exo synergism in Trichoderma reesei cellulase to increase the cellulose degrading capability. Microbial Cell Factories 2019, 18:9.

20. Mandels M, Medeiros JE, Andreotti RE, Bissett FH: Enzymatic hydrolysis of cellulose: Evaluation of cellulase culture filtrates under use conditions. Biotechnology and Bioengineering 1981, 23:20092026.

21. Gietz RD, Schiestl RH: Frozen competent yeast cells that can be transformed with high efficiency using the LiAc/SS carrier DNA/PEG method. Nature Protocols 2007, 2:1-4.

22. Curran KA, Leavitt JM, Karim AS, Alper HS: Metabolic engineering of muconic acid production in Saccharomyces cerevisiae. Metabolic Engineering 2013, 15:55-66.

23. Michielse CB, Hooykaas PJ, van den Hondel CA, Ram AF: Agrobacterium-mediated transformation of the filamentous fungus Aspergillus awamori. Nature Protocols 2008, 3:1671-1678.

24. Zhao C, Fang H, Chen S: Single cell oil production by Trichosporon cutaneum from steam-exploded corn stover and its upgradation for production of long-chain alpha,omega-dicarboxylic acids. Biotechnology for Biofuels 2017, 10:202.

25. Fang $\mathrm{H}$, Zhao $\mathrm{C}$, Chen S: Single cell oil production by Mortierella isabellina from steam exploded corn stover degraded by three-stage enzymatic hydrolysis in the context of on-site enzyme production. Bioresource Technology 2016, 216:988-995.

26. Ghose TK: Measurement of cellulase activities. Pure and Applied Chemistry 1987, 59:257-268.

27. Henry SA, Gaspar ML, Jesch SA: The response to inositol: Regulation of glycerolipid metabolism and stress response signaling in yeast. Chemistry and Physics of Lipids 2014, 180:23-43.

28. You C, Shi T, Li Y, Han P, Zhou X, Zhang YP: An in vitro synthetic biology platform for the industrial biomanufacturing of myo-inositol from starch. Biotechnology and Bioengineering 2017, 114:18551864. 
29. Meng D, Wei X, Zhang Y-HPJ, Zhu Z, You C, Ma Y: Stoichiometric Conversion of Cellulosic Biomass by in Vitro Synthetic Enzymatic Biosystems for Biomanufacturing. ACS Catalysis 2018, 8:9550-9559.

30. Fang $H$, Zhao $C$, Song $X Y$ : Optimization of enzymatic hydrolysis of steam-exploded corn stover by two approaches: response surface methodology or using cellulase from mixed cultures of Trichoderma reesei RUT-C30 and Aspergillus niger NL02. Bioresource Technology 2010, 101:41114119.

31. Zhao C, Deng L, Fang H, Chen S: Microbial oil production by Mortierella isabellina from corn stover under different pretreatments. RSC Advances 2017, 7:56239-56246.

32. Fang $\mathrm{H}$, Zhao $\mathrm{C}$, Song $\mathrm{X}-\mathrm{Y}$, Chen $\mathrm{M}$, Chang Z, Chu J: Enhanced cellulolytic enzyme production by the synergism between Trichoderma reesei RUT-C30 and Aspergillus niger NL02 and by the addition of surfactants. Biotechnology and Bioprocess Engineering 2013, 18:390-398.

33. Xu Q, Singh A, Himmel ME: Perspectives and new directions for the production of bioethanol using consolidated bioprocessing of lignocellulose. Current Opinion in Biotechnology 2009, 20:364-371.

34. Qu YN, Yan HJ, Guo Q, Li JL, Ruan YC, Yue XZ, Zheng WX, Tan TW, Fan LH: Biosynthesis of Dglucaric acid from sucrose with routed carbon distribution in metabolically engineered Escherichia coli. Metabolic Engineering 2018, 47:393-400.

35. Zhao C, Deng L, Fang H: Mixed culture of recombinant Trichoderma reesei and Aspergillus nigerfor cellulase production to increase the cellulose degrading capability. Biomass and Bioenergy 2018, 112:93-98.

36. Hollinshead W, He L, Tang YJ: Biofuel production: an odyssey from metabolic engineering to fermentation scale-up. Frontiers in Microbiology 2014, 5:344.

\section{Tables}

Table 1 Details on the bioprocesses from Avicel to D-glucaric acid.

\begin{tabular}{llllll}
\hline & SHF $^{1}$ & SSF $^{2}$ & CBP $^{3}$ & Glu $^{4}$ & G+I $^{5}$ \\
\hline te & Avicel & Avicel & Avicel & Glucose & Glucose and myo-inositol \\
sr bioprocesses (g) & & & & & \\
(g) & 50 & 50 & 15 & 0 & 0 \\
sitol (g) & 28.31 & N.A. & N.A. & 30 & 30 \\
e dosage (FPIU/ g glucan) & 0 & 0 & 0 & 0 & 10.8 \\
llulase loading (FPIU) & & & & & \\
sr cellulase production (g) & 25 & 25 & 0 & 0 & 0 \\
icel (g) & & & & & \\
:ic acid (g) & 1250 & 1250 & 0 & 0 & 0 \\
D-glucaric acid/ g total Avicel) & & & & & 0 \\
& 13.07 & 13.07 & 0 & 0 & 0 \\
& 63.07 & 63.07 & 15 & 0 & 0 \\
& 3.74 & 1.22 & 0.54 & 4.52 & 9.53 \\
& & & & & N.A. \\
\hline
\end{tabular}


${ }^{1}$ SHF, separated saccharification and fermentation by $S$. cerevisiae LGA-1.

${ }^{2}$ SSF, simultaneous saccharification and fermentation by $S$. cerevisiae LGA-1.

${ }^{3}$ CBP, consolidated bioprocessing by T. reesei Rut-C30 and S. cerevisiae LGA-1.

${ }^{4}$ Fed-batch fermentation of glucose by $S$. cerevisiae LGA-1.

${ }^{5}$ Fed-batch fermentation of glucose and myo-inositol by S. cerevisiae LGA-1.

Glu, glucose.

G+I, glucose and myo-inositol.

N.A., not applicable.

Table 2 Details on the bioprocesses from steam-exploded corn stover (SECS) to D-glucaric acid.

\begin{tabular}{|c|c|c|c|c|c|}
\hline & $\mathrm{SHF}^{1}$ & $\mathrm{SSF}^{2}$ & $\mathrm{CBP}^{3}$ & Glu $^{4}$ & $\mathrm{G}+\mathrm{I}^{5}$ \\
\hline te & SECS & SECS & SECS & Glucose & Glucose and myo-inositol \\
\hline \multicolumn{6}{|l|}{ Ir bioprocesses (g) } \\
\hline (g) & 100 & 100 & 15 & 0 & 0 \\
\hline sitol (g) & 39.73 & N.A. & N.A. & 30 & 30 \\
\hline e dosage (FPIU/ g glucan) & 0 & 0 & 0 & 0 & 10.8 \\
\hline \multicolumn{6}{|l|}{ llulase loading (FPIU) } \\
\hline Ir cellulase production (g) & 25 & 25 & 0 & 0 & 0 \\
\hline \multicolumn{6}{|l|}{$\mathrm{CS}(\mathrm{g})$} \\
\hline ic acid (g) & 2500 & 2500 & 0 & 0 & 0 \\
\hline \multicolumn{6}{|c|}{ D-glucaric acid/ g total SECS) } \\
\hline & 30.61 & 30.61 & 0 & 0 & 0 \\
\hline & 130.61 & 130.61 & 15 & 0 & 0 \\
\hline & 4.92 & 1.14 & 0.45 & 4.52 & 9.53 \\
\hline & 0.0377 & 0.0087 & 0.0300 & N.A. & N.A. \\
\hline
\end{tabular}

${ }^{1}$ SHF, separated saccharification and fermentation by $S$. cerevisiae LGA-1.

2 SSF, simultaneous saccharification and fermentation by S. cerevisiae LGA-1.

${ }^{3}$ CBP, consolidated bioprocessing by T. reesei Rut-C30 and S. cerevisiae LGA-1.

${ }^{4}$ Fed-batch fermentation of glucose by $S$. cerevisiae LGA-1.

${ }^{5}$ Fed-batch fermentation of glucose and myo-inositol by $S$. cerevisiae LGA-1.

Glu, glucose.

G+I, glucose and myo-inositol.

N.A., not applicable.

\section{Figures}




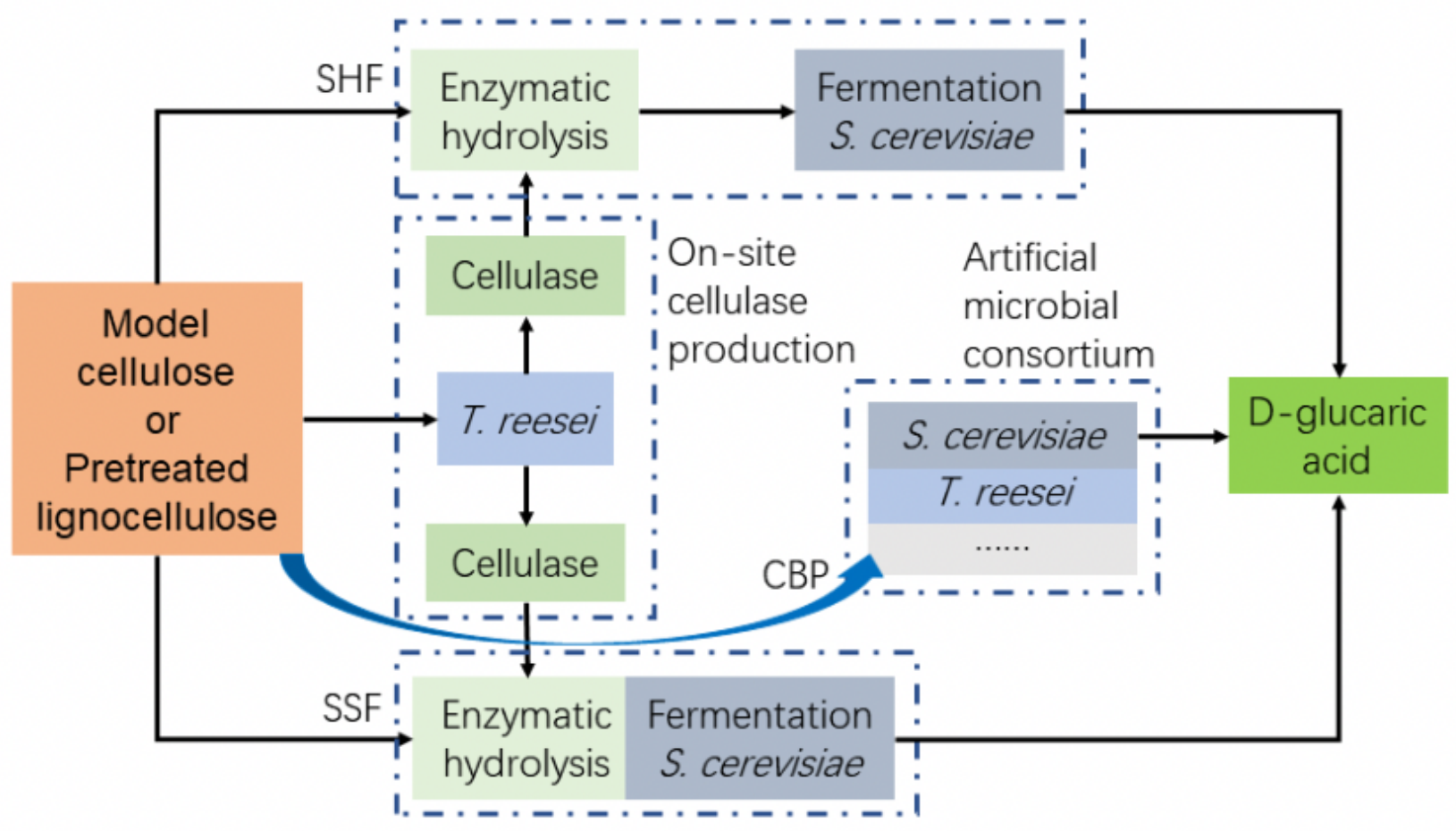

Figure 1

Diagram of biotechnological D-glucaric acid production from lignocellulose. SHF, separated hydrolysis and fermentation; SSF, simultaneous saccharification and fermentation; CBP, consolidated bioprocessing. 

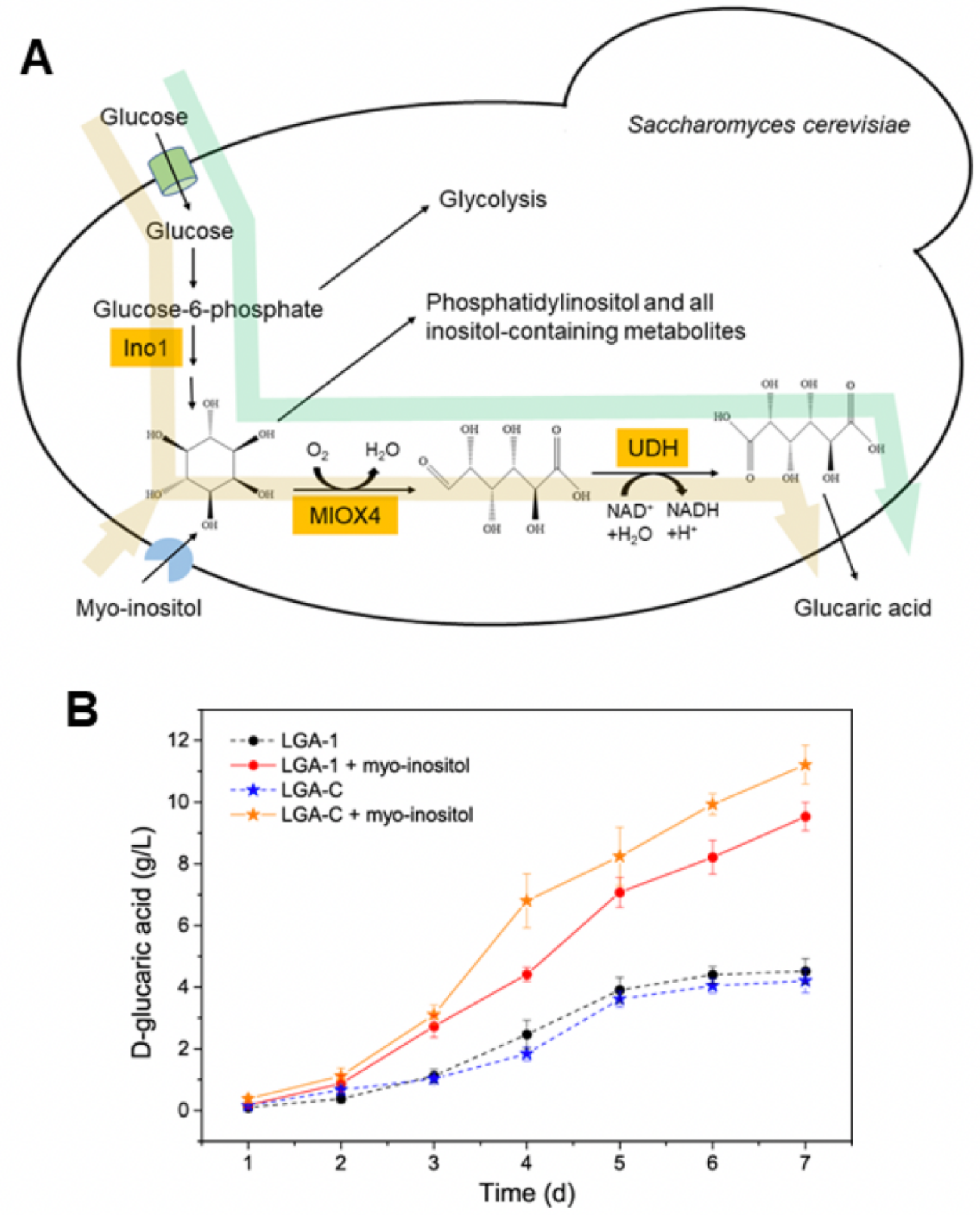

Figure 2

(A) Biosynthetic pathway of D-glucaric acid introduced into S. cerevisiae. Ino1, myo-inositol-1-phosphate synthase; MIOX4, myo-inositol oxygenase; UDH, uronate dehydrogenase. Green arrow illustrates Dglucaric acid production from glucose, and yellow one shows D-glucaric acid production from glucose and myo-inositol. (B) Time courses of fed-batch fermentations on YPD medium without and with 10.8 g/L myo-inositol. LGA-1 and LGA-C are the engineered S. cerevisiae strains capable of producing D- 
glucaric acid. Data shown here are average values of at least three biological replicates and error bars are standard deviations.
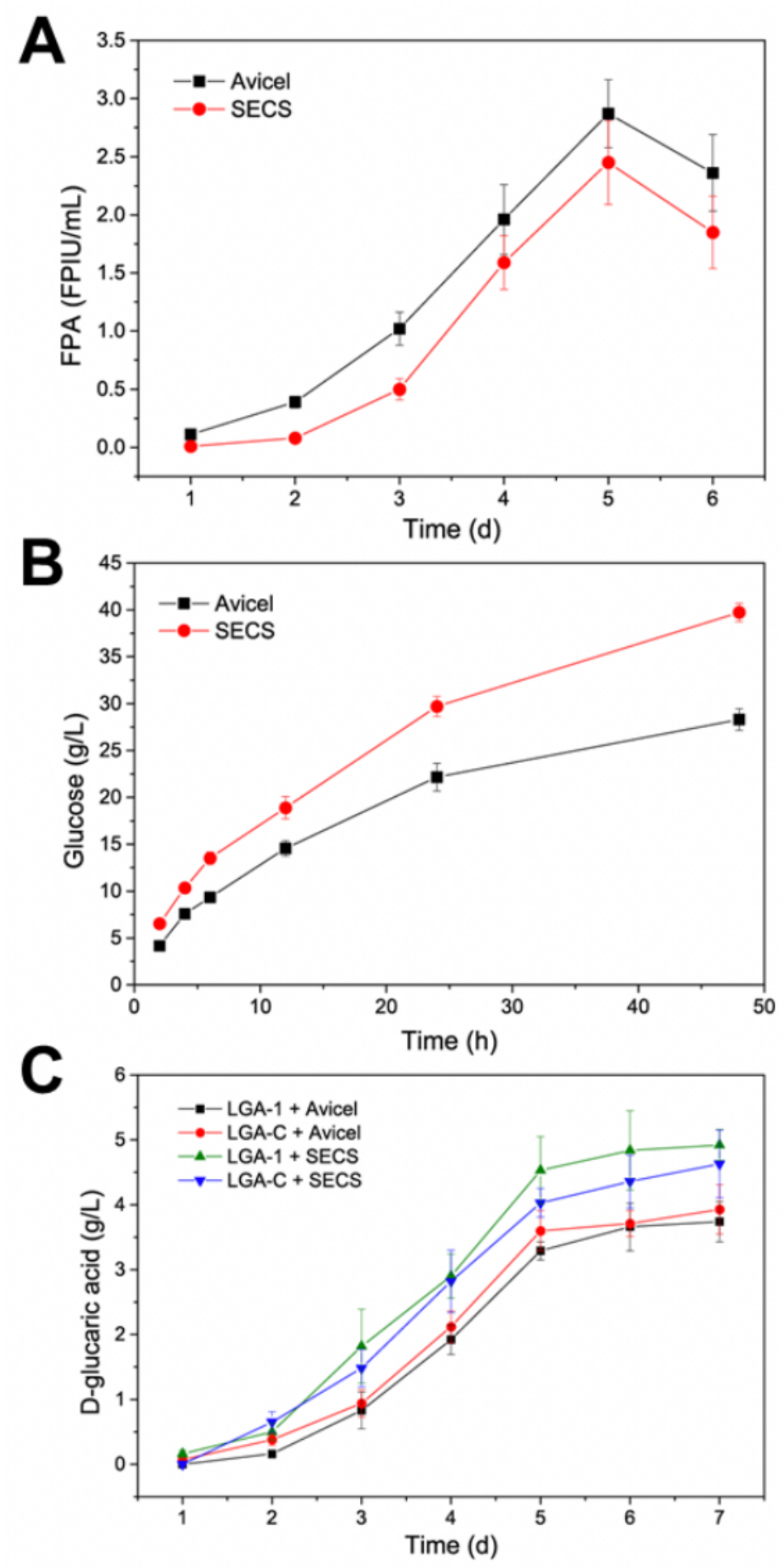

Figure 3

(A) Time courses of cellulase production by T. reesei Rut-C30 from Avicel and steam-exploded corn stover (SECS). (B) Enzymatic hydrolysis of Avicel and SECS by the cellulases produced in the context of on-site cellulase production. (C) Time courses of batch fermentations on Avicel hydrolysate and SECS 
hydrolysate. LGA-1 and LGA-C are the engineered S. cerevisiae strains capable of producing D-glucaric acid. Data shown here are average values of at least three biological replicates and error bars are standard deviations.
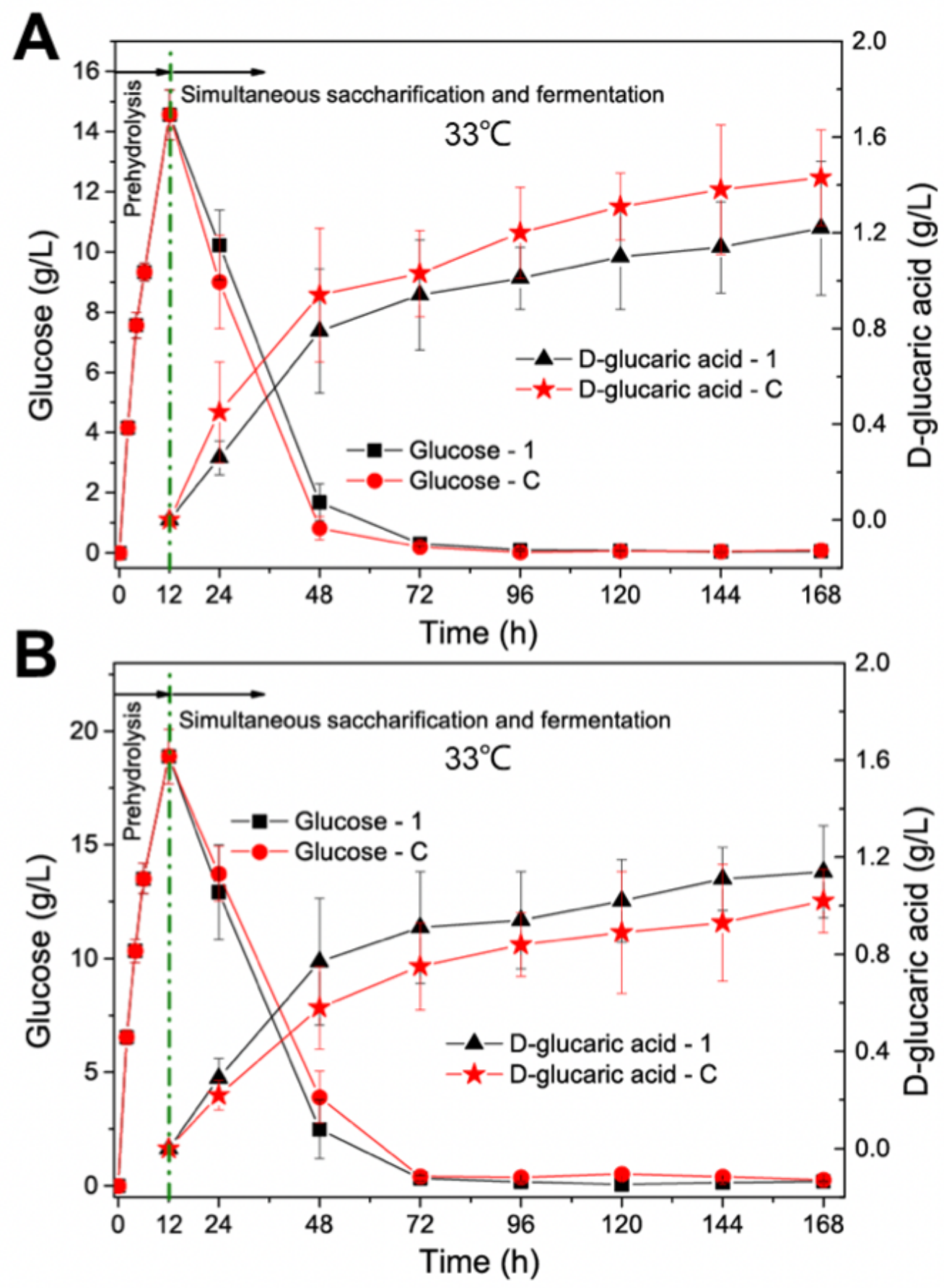

Figure 4

Simultaneous saccharification and fermentation of Avicel (A) and steam-exploded corn stover (SECS) (B) at $33^{\circ} \mathrm{C}$ from $12 \mathrm{~h}$ to $168 \mathrm{~h}$ after enzymatic prehydrolysis at $50^{\circ} \mathrm{C}$ for $12 \mathrm{~h}$. LGA-1 and LGA-C are the 
engineered S. cerevisiae strains capable of producing D-glucaric acid. Data shown here are average values of at least three biological replicates and error bars are standard deviations.
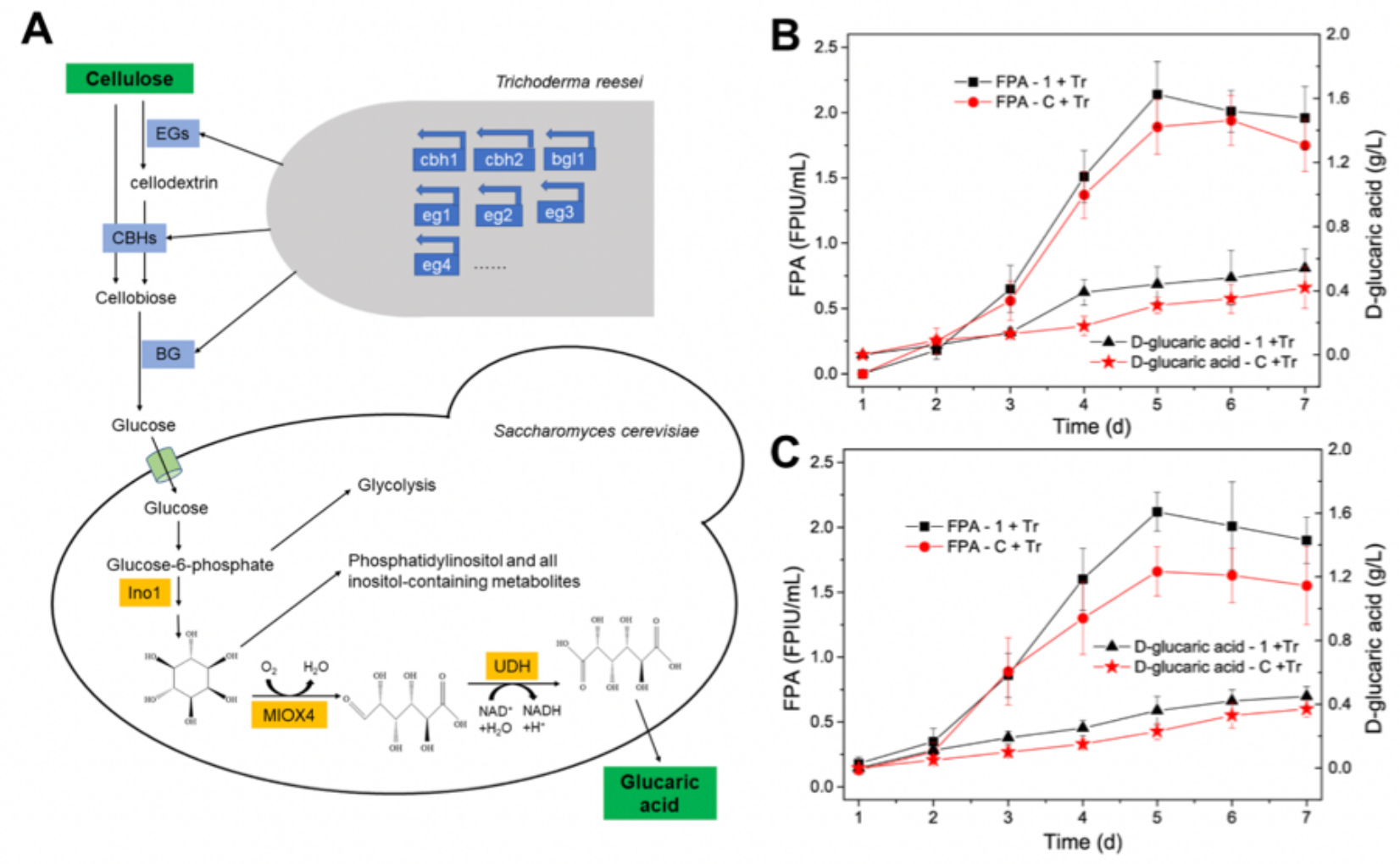

\section{Figure 5}

(A) Diagram of CBP of cellulose for D-glucaric acid production by the microbial consortium consisted of T. reesei Rut-C30 and S. cerevisiae LGA-1. EGs, endoglucanases; CBHs, cellobiohydrolases; BG, $\beta$ glucosidase; Ino1, myo-inositol-1-phosphate synthase; MIOX4, myo-inositol oxygenase; UDH, uronate dehydrogenase. (B) FPAs and concentrations of D-glucaric acid during CBP of Avicel. (C) FPAs and concentrations of D-glucaric acid during CBP of steam-exploded corn stover (SECS). 1 and C in legends stand for S. cerevisiae LGA-1 and LGA-C, respectively. Tr in legends stands for T. reesei Rut-C30. Data shown here are average values of at least three biological replicates and error bars are standard deviations. 

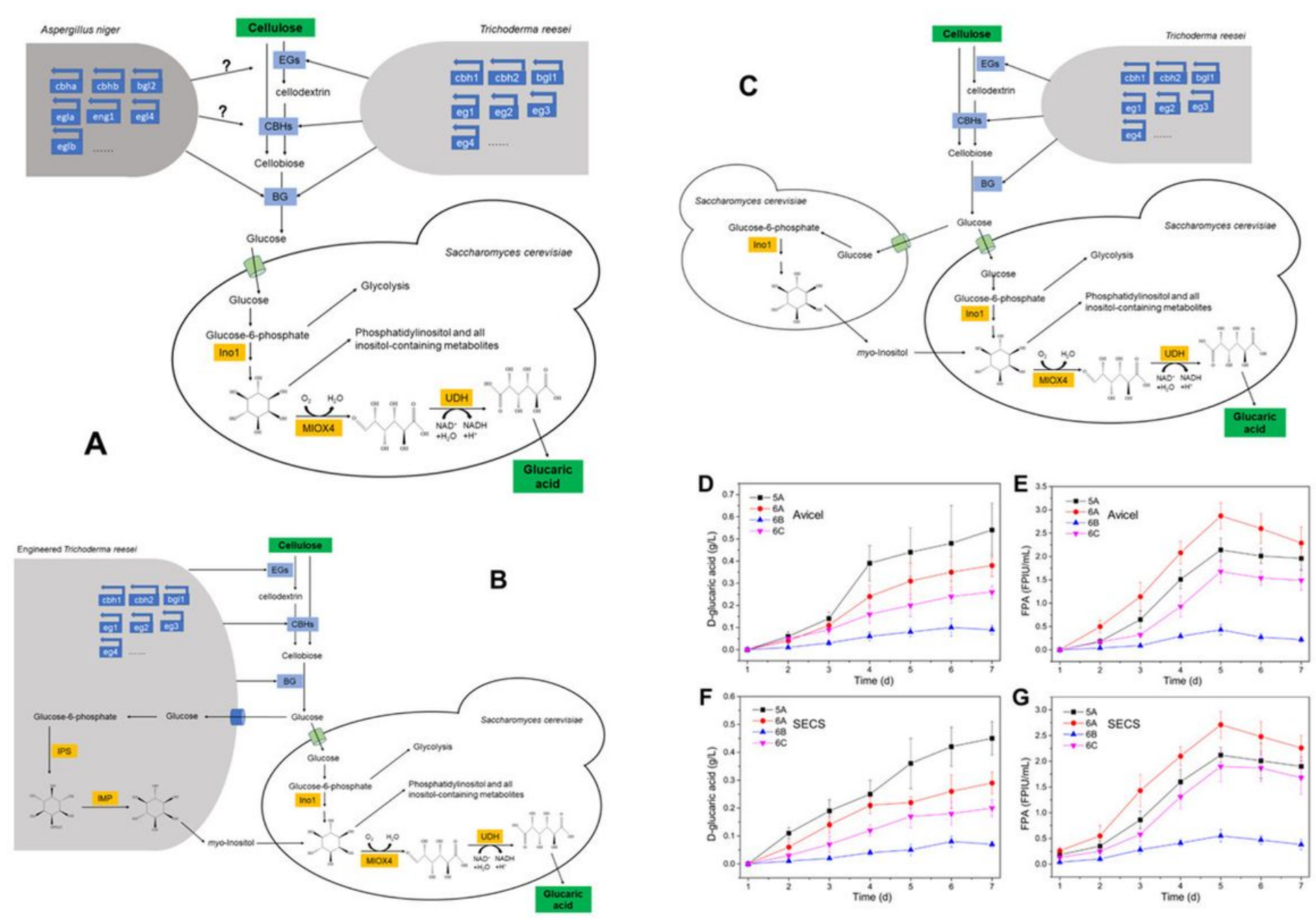

\section{Figure 6}

(A) Diagram of CBP of cellulose for D-glucaric acid production by the microbial consortium consisted of T. reesei Rut-C30, A. niger CICC2103 and S. cerevisiae LGA-1. (B) Diagram of CBP of cellulose for Dglucaric acid production by the microbial consortium consisted of the engineered $\mathrm{T}$. reesei and $\mathrm{S}$. cerevisiae LGA-1. (C) Diagram of CBP of cellulose for D-glucaric acid production by the microbial consortium consisted of T. reesei Rut-C30, S. cerevisiae $\triangle \mathrm{opi} 1$ and S. cerevisiae LGA-1. Concentrations of D-glucaric acid (D) and FPAs (E) during CBP of Avicel. Concentrations of D-glucaric acid (F) and FPAs (G) during CBP of steam-exploded corn stover (SECS), 5A, 6A, 6B and $6 \mathrm{C}$ in the legends of Fig. $6 \mathrm{D}, \mathrm{E}, \mathrm{F}$ and $\mathrm{G}$ represents the CBPs illustrated in Fig. 5A, 6A, 6B and 6C, respectively. Data shown here are average values of at least three biological replicates and error bars are standard deviations.

\section{Supplementary Files}

This is a list of supplementary files associated with this preprint. Click to download.

- FigureS1.docx

- Figures2.docx 
- FigureS3.docx

- Figures4.docx

- FigureS5.docx

- Figures6.docx

- FigureS7.docx

- Figures8.docx

- TableS1.docx

- Tables2.docx 